\section{The epidemiology, evaluation, and assessment of lateral ankle sprains in athletes}

\author{
G Javier Cavazos Jr. ${ }^{1 *}$ and Lawrence B Harkless ${ }^{2}$ \\ ${ }^{1}$ President, Elect/Fellow, American College of Podiatric Medicine, Fellow of Faculty, Royal College \\ Physicians and Surgeons, Glasgow, Scotland \\ ${ }^{2}$ Interim Dean, School of Podiatric Medicine, Professor Department of Surgery, University of Texas \\ Rio Grande Valley (UTRGV) School of Medicine, USA
}

\section{Summary}

Approximately 30,000 ankle injuries occur every day in the United States. With the incidence estimated at more than 3 million a year and at a rate of 2.15/1,000 in the U.S. alone, medical specialists and other healthcare providers caring for the foot and ankle must take notice. Despite the millions of ankle injuries sustained annually, the true incidence may be underestimated, as fewer than half of individuals with ankle sprains seek medical attention from healthcare professionals. The economic burden associated with the evaluation, diagnosis, and treatment is close to $\$ 4$ billion annually. Ankle sprains account for half of all sports injuries and remains a difficult diagnostic and therapeutic challenge in the athlete. Accurate diagnosis is critical as $40 \%$ of ankle sprains are misdiagnosed or poorly treated leading to chronic ankle pain and disability. Implementing evidence supported diagnostic and treatment strategies is the goal for ensuring safe and rapid return to play.

The Lateral Ankle Sprain (LAS) is among the most common type of ankle sprains suffered during athletic activities. Up to $80 \%$ of LAS are of the inversion type, and $75 \%$ lead to recurrence and instability. Although most individuals experiencing a LAS return to activity within six weeks, many report continued pain, diminished function, and instability.

The purpose of this review is to highlight the epidemiology, pathoetiology, pathoanatomy, and biomechanics of the LAS, enabling sports physicians to implement the best practice guidelines and protocols to manage this common enigma.

\section{More Information}

*Address for Correspondence:

G Javier Cavazos Jr., DPM, FACPM, FFPM RCPS, President, Elect/Fellow, American College of Podiatric Medicine, Fellow of Faculty, Royal College Physicians and Surgeons, Glasgow, Scotland,

Email: javiercavazosdpm@gmail.com; javnancjon@aol.com

Submitted: May 03, 2021

Approved: May 25, 2021

Published: May 26, 2021

How to cite this article: Cavazos GJ Jr., Harkless LB. The epidemiology, evaluation, and assessment of lateral ankle sprains in athletes. J Sports Med Ther. 2021; 6: 008-017.

DOI: 10.29328/journal.jsmt.1001052

Copyright: (c) 2021 Cavazos GJ Jr., et al. This is an open access article distributed under the Creative Commons Attribution License, which permits unrestricted use, distribution, and reproduction in any medium, provided the original work is properly cited.

Check for updates

OPEN ACCESS

\section{Introduction}

The Lateral Ankle Sprain (LAS) represents one of the most common musculoskeletal injuries seen in podiatric, primary care and orthopedic clinics throughout the United Sates [1-3]. Often underestimated and mismanaged, LAS are not benign injuries and often result in delayed treatment, prolonged recovery times, and long term sequela. An estimated 30,000 ankle injuries occur in the US each day, translating into an enormous burden on the health care industry, with millions of dollars being spent on treatment each year. Moreover, sports related activities attribute to a majority of all ankle injuries. It is estimated that upward of $45 \%$ of all sports related injuries involve the ankle and associated structures [4-6]. Ankle injuries can become serious, and should be given immediate attention and care. Ankle sprains can lead to a significant amount of pain, limited mobility, and disability which in turn can lead to depression and a poorer quality of life. While a history ankle sprain, ankle instability and ligamentous laxity make an athlete at risk for injury, a previous sprain is the most common risk factor predicting a recurrent injury. The athlete; professional and amateur, who continually exert their bodies to their limits are most susceptible to this injury of the lower extremity. Recovery time and safe return to the playing field is a frequent concern of all athletes. The rates of recurrence, the condition of symptoms, and the rising cost of evaluation and management alarm physicians, as more efficient methods, for diagnosis and treatment are explored. Because ankle sprains are common, and disabling to athletes, healthcare professionals must be able to implement the most current and evidence supported treatment strategies to ensure safe and rapid return to play $[30-32,34,35,38]$.

\section{Epidemiology}

It is estimated that in the U.S., 3 million people with ankle 
injuries visit the Emergency Department(ED) every year.7,8 Ankle injuries comprise $10 \%$ of all ED visits; $85 \%$ being the inversion type, and the anterior talofibular ligament (ATFL ) being afflicted approximately 65 percent of the time [4].

A review of ED records in the U.S. estimate the incidence rate of an ankle sprain to be 2.15 per 1000 persons, with the highest age cohort being between the ages of 15 and 19 (7.2 per 1000 person-years). Patients with ankle sprains are typically young and physically active. Males between the ages of 14 and 24 and females over the age of 30 have a higher incidence rate compared to their respective counterparts $[3,9]$. The true incidence of LAS is likely greater than estimated because fewer than half of all individuals with ankle sprains seek care from any medical professional $[5,10]$.

The prevalence of sports related ankle injuries range from $20 \%$ to $50 \%$. According to the American Academy of Orthopedic Surgeons, 25\% of athletic injuries are foot and ankle related. Athletes involved in sports that require jumping and running are typically at higher risk. Sports activities like running, basketball, soccer, football, dancing and martial arts place considerable performance demands on the foot and ankle and are commonly associated with injuries. Stefano and Padua approximated $20 \%-40 \%$ of all athletic injuries involved ankle sprains.11 Fong systematically reviewed 227 sports injury studies, including 201,600 patients from 70 different sports and 38 countries. Fong, et al. found the ankle joint to be the most commonly injured area (34\%) of the entire body.

When comparing different sports and activities, the incidence rates of ankle sprains was highest amongst athletes playing field hockey, followed by volleyball, football, basketball, cheerleading, ice hockey, lacrosse, soccer, rugby, track and field, gymnastics, and softball [12]. In a separate study, Waterman reported athletic-associated ankle sprains were most common in basketball (41.1\%), football $(9.3 \%)$, and soccer $(7.9 \%)$ [3].

When comparing age groups and gender, Doherty reviewed 181 prospective studies and found a higher incidence of ankle sprains in females compared with males, in children compared with adolescents, and adolescents compared with adults. The sport category with the highest incidence of ankle sprain were indoor and court sports with a cumulative incidence rate of 7 per 1000 exposures.

Re-injury rates and long term sequela from ankle injuries is widely reported in the literature. The rate of reinjury after a LAS is noteworthy. A systematic review of studies evaluating re-injury rates in LAS revealed recurrent injury between $3 \%$ and $34 \%$. The time interval between initial injury and a second injury varied greatly, ranging from within 2 weeks to 96 months $[14,15]$. A recent prospective study on track-andfield athletes found a 17\% reinjury rate within 2 years [16].
Reinjury rates, however, may be greater in high-risk sports such as basketball, where recurrent LAS rates of $73 \%$ have been reported $[10,12,17,30,31,38]$.

Lateral ankle sprains are not benign injuries. As many as 4 in 10 individuals will develop chronic ankle instability (CAI) after a first-time sprain [11]. Over $50 \%$ of adults with osteoarthritis (OA) of the ankle, report a history of sportrelated ankle injury, $85 \%$ of whom claim a previous history of LAS.12 Braun reported between $55 \%-72 \%$ of those with LAS having residual symptoms after 6 months, while Anandacoomarasamy \& Barnsley reported chronic instability and a lower general health quality of life 2 years after injury $[24,25,30,38]$.

The risk factors for acute lateral ankle sprain are categorized as being intrinsic or extrinsic. Intrinsic factors include the history of previous sprains, age, gender, height, weight, and body mass index. Moreover, musculoskeletal characteristics like balance, proprioception, range of motion, strength and anatomic foot type may influence a predilection for sprains. Extrinsic risk factors include the use of external bracing, type of sport being played, level of competition, and participation in neuromuscular training [9].

There is some suggestion in the orthopedic literature that $80 \%$ of patients will fully recover from an ankle sprain with non-surgical care. In this authors opinion, successful rehabilitation of a sports related ankle sprain is dependent on appropriate evaluation and diagnosis, timely intervention, aggressive non- surgical care centered on protection and offloading the injury, early optimal loading, edema control, rehabilitation and prevention of recurrence.

The purpose of understanding the epidemiology of ankle sprains is to better comprehend predisposing risk factors, and clinical patterns that will aid with early detection, diagnostic criteria, management options and long term prognosis.

\section{Anatomy}

The ankle joint is a hinged synovial joint that is formed by the articulation of the talus, tibia, and fibula. Together, the three osseous structures form the ankle mortise. The ankle joint is intrinsically stable due to the anatomic integrity of the distal tibia, which forms the roof and medial wall the ankle, and the distal fibula which forms the lateral wall of the joint (Diagram 1). The talus has limited motion in the frontal plane and uninhibited motion in the sagittal plane. The talar dome is wider anteriorly and upon dorsiflexion, the talus fits tightly in the mortise (Diagrams 2,3). Plantarflexion of the talus increases ankle range of motion in the sagittal plane. The ankle joint is secured by ligaments on the medial and lateral aspect of the ankle joint and by the joint capsule. The lateral collateral ligaments are inherently weaker than their medial counterparts and will be subjected to more stress during gait. The position of the talus, the integrity of the ligaments along with the stress applied explain why lateral ligament injury is more common than injury to the medial ankle (Diagram 4). 


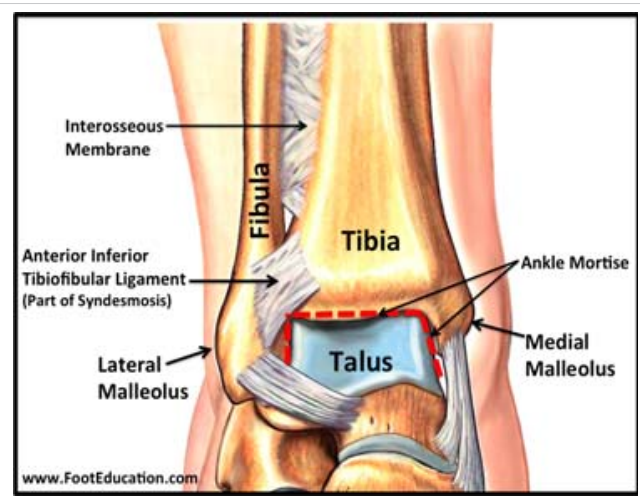

Diagram 1: Anterior view of the ankle: Tibia Fibula and Ankle Mortice. Ref: Foot Education.com

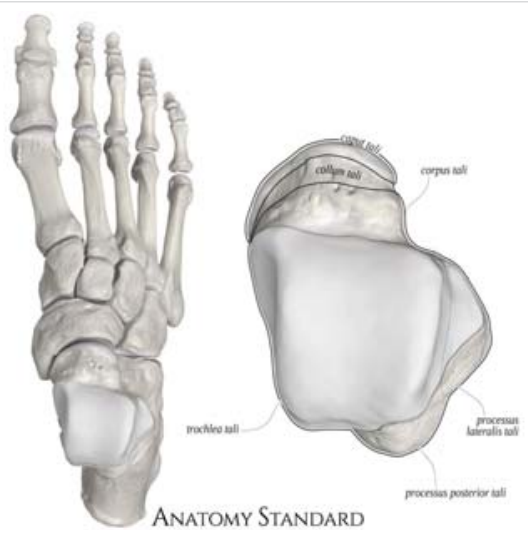

Diagram 2: Superior View of Talus. Ref: Anatomy Standard.

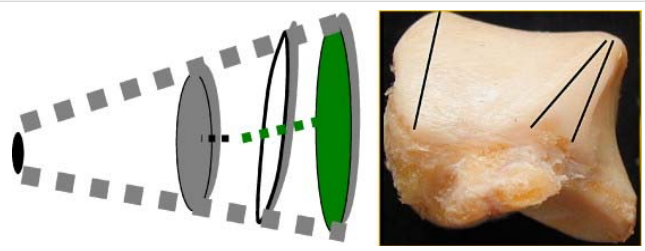

Diagram 3: Shape of talus; Conelike structure wider anteriorly. Ref: Foot Education.com

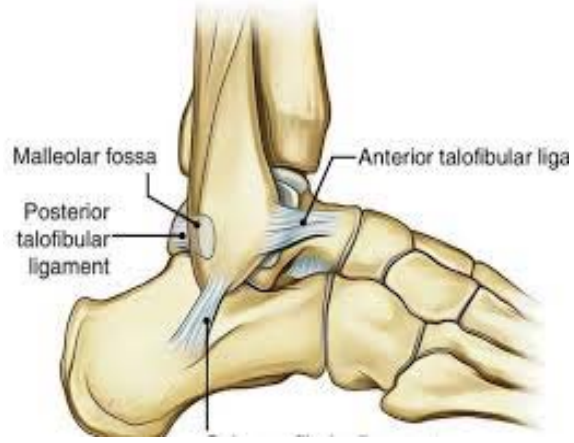

Diagram 4: Lateral view of the ankle collateral ligaments. Foot Education.com

The lateral collateral ligaments include the anterior talofibular ligament (ATFL), the posterior talar fibular ligament (PTFL), and the calcaneofibular ligament (CFL). The ATFL is formed by two distinct bands separated by a slight gap that allows the penetration of vascular branches. The ATFL originates from the inferior oblique segment of the anterior border of the lateral malleolus, it courses anterior- medially and inserts on the talar body just anterior to the lateral malleolus articular surface. The ATFL is an intracapsular ligament which is relaxed upon dorsiflexion and tightened during plantarflexion

The calcaneofibular ligament (CFL) is usually the second ligament injured during an inversion injury originating from the lower segment of the interior border of the lateral malleolus. The CFL is a wide band structure that courses posteriorly, inferiorly, and medially, and inserts on a small tubercle located on the posterior aspect of the lateral calcaneal surface. The CFL is extracapsular ligament but opposing the ATFL, the CFL is relaxed on plantar flexion and tightens with dorsiflexion.

The posterior talofibular ligament (PTFL) originates on the medial surface of the lateral malleolus from the lower segments of the digital fossa. The PTFL courses horizontally towards the lateral and posterior aspect of the talus which inserts on the posterior lateral tubercle of the talus. The PTFL is an intracapsular ligament that becomes more vertical upon dorsiflexion and prevents the talus from slipping backwards [18-20,31,32].

\section{Pathoetiology}

The correlation between the anatomy and biomechanics of the ankle joint is crucial to understanding the pathomechanics and etiology of the Lateral Ankle Sprain (LAS). The position of the talus relative to the long axis of the leg, and the alignment of the lateral collateral ligaments relative to the axis of the tibia helps determine the function of the three lateral ligaments and their susceptibility to an inversion ankle injury. When the ankle joint is in neutral dorsiflexed position, The ATFL is perpendicular to the axis of the tibia and the CFL is parallel, providing resistance to inversion or a varus tilt. When the Talus is plantarflexed (most common position during lateral ankle inversion injuries), the ATFL is parallel to the axis of the tibia and the CFL is perpendicular. The ATFL in this position resists inversion stress. When the foot plantarflexes from the ankle, the ATFL is parallel to the axis of the tibia, will tighten and stretch with STJ inversion while the CFL becomes more relaxed. The stress applied on the ATFL, in this subtalar joint supinated position, will determine the extent of the injury and tearing of the ATFL, CFL and other associated structures [18-20] (Diagrams 5-7).

During an inversion ankle injury, closed kinetic supination is demonstrated by inverting the foot relative to the ground coupled with external rotation of the lower leg. The torque force on the ankle will correspond to the extent of plantarflexion of the talus, which will alter the axis of the ATFL and CFL straining them to their limits [18-20].

\section{Mechanism of Injury}

The inversion ankle sprain is one of the most common 


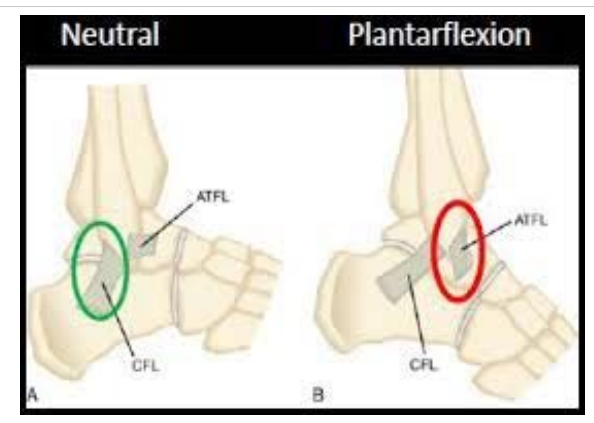

Diagram 5: ATFL alignment with Dorsiflexed and Plantarflexed Ankle. Ref: Sdapa.net

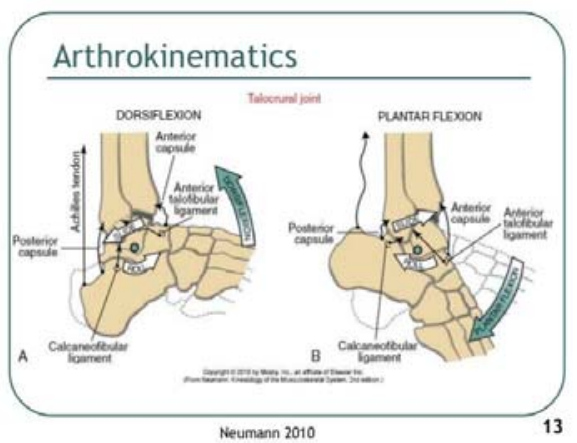

Diagram 6: Biomechanics of the Ankle. Ref: Newman, Kinesiology of the Musculoskeletal System.2010.

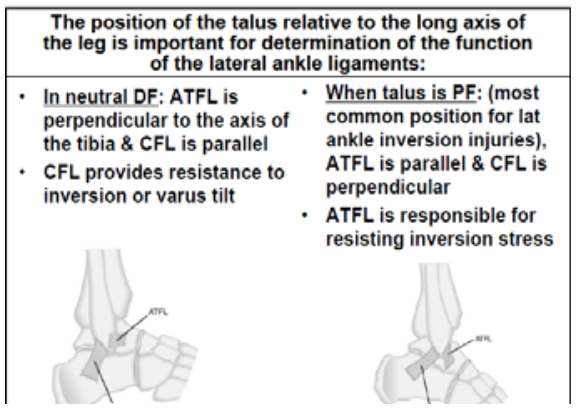

Diagram 7: Position of ATFL to axis of Tibia. Ref: Five Common Sports Injuries: Timothy L. Miller, MD Assistant Professor OSU Orthopedic Surgery and Sports Medicine. The Ohio State University Wexner Medical Center.

injuries in all of sports. An inversion ankle injury occurs with supination (plantarflexion, inversion, and internal rotation of the foot) coupled with external rotation of the lower leg. An inversion ankle sprain is depicted when the foot rolls inward, the ankle rotates outward, and the lateral ankle muscles, tendons and ligaments are stretched beyond their normal limit. Specifically, the anterior talofibular ligament (ATFL) reported to be the weakest of the collateral ligaments, becomes the first ligament injured and or ruptured [4]. Tearing of the ATFL is then followed by strain, tear or complete rupture to the calcaneofibular (CFL), and posterior talofibular (PTFL) ligaments respectively. Isolated injury to the ATFL occurs in $66 \%$ of LAS, while the ATFL and CFL rupture simultaneously $20 \%$ of the time. The PTFL is not commonly injured due amount of supination force required by the foot in an inversion sprain or the amount of ankle dorsiflexion required to strain or tear the ligament $[18,19]$ (Diagram 8).

\section{Grading lateral ankle sprains}

Acute lateral ankle sprains are often categorized by the severity of the injury. A grading scale ranges from no significant structural injury, to complete rupture of the ligamentous structures. Traditionally, lateral ankle sprains are graded I, II, and III, representing the extent and severity of ligament damage, with a grade I being the least and grade III being the most severe type of injury (Diagram 9). Several lateral ankle-ligament grading systems have been described in the medical literature making critical appraisal of a study difficult. Karlsson's grading system focuses on a single ligament; the (ATFL). A grade I injury represents a microscopic injury without altering the ligament on a macroscopic level. A grade II injury involves macroscopic stretching of the ATFL, but the ligament remains intact. A grade III injury is a complete rupture of the ATFL [26]. A downside to the Karlsson classification is only the status of the ATFL is evaluated and ignores injury to either the CFL or PTFL.

Other authors have graded lateral ankle-ligament sprains by the number of ligaments injured [19,27-29,31,32]. Three such classification systems include the O'Donahue, Leach and the AMA systems listed below:

\section{O’Donahue Classification:}

Grade I: Single tear ATFL

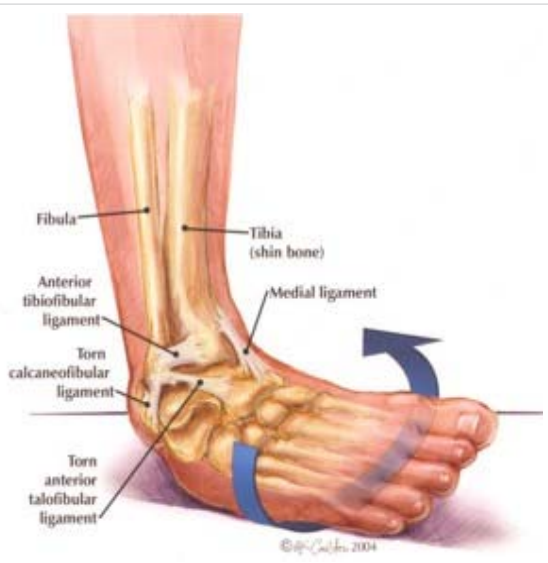

Diagram 8: Mechanism of Injury Inversion Ankle Sprain. Ref: Pinterest http://us4. campaign-archive2.com/?u=d7354354bb36100270d098153\&id=e916a487f8

\begin{tabular}{|c|c|c|}
\hline $\begin{array}{l}\text { TYPE OF } \\
\text { INJURY }\end{array}$ & $\begin{array}{l}\text { EXTENT OF } \\
\text { INJURY }\end{array}$ & $\begin{array}{l}\text { PHYSICAL } \\
\text { FINDINGS }\end{array}$ \\
\hline Grade I & $\begin{array}{l}\text { Stretch of the } \\
\text { ligament with } \\
\text { microscopic but not } \\
\text { macroscopic tearing }\end{array}$ & $\begin{array}{l}\text { Minor swelling but no } \\
\text { joint instability }\end{array}$ \\
\hline Grade II & $\begin{array}{l}\text { Involves partial tearing } \\
\text { of the particular } \\
\text { ligament }\end{array}$ & $\begin{array}{l}\text { Minor to moderate } \\
\text { swelling and some } \\
\text { instability of the } \\
\text { affected ankle }\end{array}$ \\
\hline Grade III & $\begin{array}{l}\text { Involves complete } \\
\text { rupture of the } \\
\text { ligament }\end{array}$ & $\begin{array}{l}\text { Significant swelling, } \\
\text { tenderness, and } \\
\text { ecchymosis; } \\
\text { instability of the joint }\end{array}$ \\
\hline
\end{tabular}

Diagram 9: Lateral Ankle Sprain Grading Scale. Ref: Published in Clinics in sports medicine 1988 Foot and ankle injuries. J. D. Castillo. 
Grade II: Partial tear ATFL and CFL

Grade III: Complete Tear ATFL, CFL

Leach (Anatomical) Classification:

Grade I: Tear of ATFL

Grade II: Tear of ATFL, CFL

Grade III: Tear of ATFL, CFL, PTFL

The AMA Classification:

Grade I: Stretch or Microscopic Tear of the ATFL.

Grade II: Tear of ATFL.

Grade III: Complete Tear of ATFL and CFL.

The AMA grading scale allows clinicians to recognize early strains and microtears of the ATFL that otherwise may not be considered with other systems. The AMA classifies a grade I sprain, as a mere stretching or microtear of the ATFL that can easily be detected through ultrasonography. This classification allows for early recognition and timely intervention, hence making it less likely to underestimate the potential consequences of not aggressively addressing this ubiquitous injury.

Grading systems that classify LAS will help with assessment and management, by aiding the physician establish protocols for protection, immobilization, early mobilization and recovery. Moreover, timelines for recovery and rehabilitation that range from 1 to 2 weeks (Grade I) to 6 to 10 weeks (Grade III) will help establish realistic time frames for recovery and return to activity.

\section{Clinical evaluation and assessment}

The evaluation of the LAS begins with a thorough History and Physical Examination. Eliciting the mechanism and previous history of injury, combined with astute clinical observation and palpation, will provide important insight into determining the anatomical structures affected in a patient suspected of having an ankle sprain. Thorough observation of the foot, ankle, and lower leg on the injured side will provide clarity as to which anatomic structures may be injured. The clinician should look for deformity, swelling, and discoloration [1]. As previously stated, a history of ankle sprain is the most common predisposing factor for a recurrent ankle injury, and supination of the foot ( plantarflexion and inversion) coupled with external rotation of the lower leg describes the most common mechanism of injury for a lateral ankle sprain $[1,4]$.

The examination of the Lateral Ankle Sprain (LAS) will help determine the severity of injury, grade of ligament damage, degree of stability, and a reduction in range of motion, strength, and proprioception. Moreover, an accurate assessment includes identifying any additional or associated injuries like an avulsion fracture, neurovascular injury and compression syndromes. No single exam can provide a conclusive answer, but a comprehensive evaluation will helps build a case allowing clinicians to utilize their professional judgment and experience to make a diagnosis [1,7,27,31,32].

Making an accurate diagnosis begins by reviewing the patients' medical record and history of present illness. Old medical records and previous x-rays from the ED should be reviewed. Any previous treatments provided in the Emergency Department or by another physician should be taken into account. The nature of the injury is a narrative of the mechanism of injury. A patient may not recall the specifics of an inversion sprain, but by asking the patient to carefully recreate how the injury happened may shed light as to the mechanism of occurrence and biomechanical forces applied during the injury. Pertinent questions to ask should include:

- Did the ankle roll outward or inward?

- Did the injury make you fall forward, backwards or sideways?

- What sports activity was being performed when injury occurred?

- Were you tackled from behind pinning your foot underneath?

- Did you fall from a certain height and roll out your foot?

- Describe the terrain or sports field when the injury occurred? (Grass, Dirt track, Hard Court, Sidewalk, Hills, Gravel)

- Did you step in a hole?

- Did you hear a popping sound around your ankle? (A noise, a crack, or a pop could be indicative of ligaments tearing or bones breaking)

- Are you able to move the foot and ankle

- Was the injury severe enough to take you to the ground?

- Were you able to bear weight immediately?

- Did you have the ability to continue to play after the injury or were you sidelined?

- Describe the timing of the injury by recalling when it occurred? (acute vs. chronic)

- Did you seek medical attention? What was done, when and how effective?

- Any immediate interventions? (Rest, Ice, Compress, Elevate, Offload)

- Was there any delay in medical attention and treatment?

- Any previous interventions by the emergency room doctor or primary care physician? 
The timing of the injury and its appearance immediately after the injury will help clarify the stage of inflammation and severity of the injury:

- Any pain, swelling, and most importantly bruising immediately following the injury? (Ecchymosis is usually an indicator of vascular extravasation suggestive of tissue tear and bleeding)

- Was the pain sudden, onset, or gradual? (athletes adrenaline may prevent an accurate measure of pain especially immediately after the injury occurred)

- Was the swelling sudden onset, or gradual? (sudden swelling often indicates bleeding into the joint rather than a gradual increase in synovial fluid within the joint)

The physical examination should begin with observation for gross abnormalities, edema, ecchymosis, neurovascular assessment, and palpation for areas of tenderness. Examine the vascular status by palpating the dorsalis pedis, posterior tibial, common peroneal and popliteal arteries. Sub-capillary refill time to the digits should also be assessed. Neurological exam should include eliciting for epicritic sensation, deep tendon reflexes and sensation to light touch. Severe pain or paresthesia's may be indicative of severe neurological damage or compression syndrome. Edema may be quantified using a tape measure to make a figure-8 measurement that encompasses the medial malleolus, lateral malleolus, navicular, and base of the fifth metatarsal $[1,7,21,27$,$] . Significant edema$ surrounding the entire lower leg and forefoot may suggest a more serious compression syndrome, ankle dislocation or LisFranc Injury. The presence of ecchymosis may be suggestive of vascular extravasation from a bone fracture or ligament, tenon or retinaculum tear.

Palpation for regions of tenderness over soft tissue and bony structures should include the anterior talofibular ligament (ATFL), calcaneal fibular ligament (CFL), posterior talofibular (PTFL), anterior inferior tibiofibular ligament (AITFL), syndesmosis ligaments, deltoid ligaments, extensor and flexor(Peroneal)retinaculum, peroneal, Achilles and posterior tibial tendons, Lis Franc Joint, Anterior Ankle Capsule, Lateral and Medial Malleolus, Anterior Edge of the Fibula, Base of the $5^{\text {th }}$ Metatarsal and Navicular Tuberosity [2].

Ankle ROM should be assessed carefully and passively initially to gauge the extent of the injury. Active, and resistive ROM in both the sagittal (plantar-dorsiflexion) and frontal (inversion-eversion) planes is performed only if tolerable and gradually in order to prevent further tissue damage. Limited ROM may be present in the case of an intra-articular lesion or swelling within the joint.

The Ottawa Ankle and Foot Rules provide clinical guidelines to help determine if radiography is indicated at the time of the ankle sprain. Reexamination three to five days after injury, when pain and swelling have improved, may help with the diagnosis. The Ottawa guideline describing the indications for radiography include:

1. Tenderness while palpating over the medial malleolus, lateral malleolus, navicular, and/or base of the fifth metatarsal.

2. Inability to bear weight immediately following injury or during the clinical evaluation.

3. Tenderness that extends $6 \mathrm{~cm}$ superiorly from the tip of either malleolus.

\section{Pain to the midfoot (Lis Franc Joint) $[1,2,22,23,31,32]$.}

Radiographs should be performed immediately if gross abnormalities are visualized. Standard radiographic views include anterior to posterior, lateral, and ankle mortise. Even if radiographs are inconclusive, a Salter-Harris type 1 fracture should be considered in a skeletally immature patient if palpation elicits tenderness over the distal tibia or fibula growth-plates [1,22].

\section{Special tests}

Clinical stability tests to assess ligamentous disruption of the collateral ligaments are best performed between 4 and 7 days after the injury, when the acute pain and swelling are diminished and the patient is able to relax during the examination $[1,9,21,27]$. The anterior drawer test and the talar tilt test are specific for assessing the integrity of the ATFL, and the CFL respectively.

Anterior drawer test: The Anterior Drawer Test is the most common test performed to assess the integrity of the ATFL. Sensitivity values have been reported from 32\% - 80\% while the specificity for the exam has been reported as high as 80\% [1]. Moreover, an Anterior Drawer Positive test performed five days after injury is more sensitive and specific than an examination performed within 48 hours of the injury [1]. The anterior drawer test, also referred to as the push pull test, is usually performed under anesthesia. AD tests to the unaffected limb is also recommended to avoid a false positive result. With the patient seated knee flexed, and the ankle hanging off the table, the examiner stabilizes the distal tibia with one hand, and grasps and pulls the foot forward with the other hand. The AD test is best performed in neutral dorsiflexion to evaluate the CFL, and in ankle plantarflexion to assess the integrity of the ATFL. There should be minimal movement. A movement differential compared to the unaffected contralateral side is suggestive of ATFL compromise. A $3.5 \mathrm{~mm}$ translational displacement and an anterior dimple seen clinically is considered a positive test for an ATFL tear $[1,6,7,9,27]$.

Talar tilt test: The Talar tilt test is usually performed under anesthesia and requires simultaneous radiographic 
imaging. Bilateral evaluation is recommended to prevent a false positive result. The patient is seated, leg secured with examiners open hand and heel grasped from behind with the opposite hand. A varus inversion force is applied to produce a talar tilt. The test is performed with the ankle in a neutral dorsiflexed and plantarflexed position. A greater than $10^{\circ}$ differential between the affected ankles to the uninjured one is considered pathological. Lauren et al. reports physiological asymmetry rarely exceeds $10^{\circ}$ while Anderson found a greater than $15^{\circ}$ difference indicative of ATFL and CFL rupture $[1,6,7,27]$.

Cotton test: The cotton test is utilized to help determine the presence of a high ankle sprain occurring when the athlete is pushed or tackled from behind, pinning the foot and ankle in a plantarflexed position, stressing the anterior ankle capsule and ligaments. This test assesses the integrity of the distal tibiofibular ligaments, and is performed by translating the talus within the mortise from medial to lateral in a neutral position. Any increased translation or pain may suggest syndesmosis involvement $[7,27]$.

Squeeze test: This squeeze test assesses the integrity of the distal tibiofibular ligaments. It is performed by squeezing the proximal third of the leg firmly enough to cause a reciprocal splaying at the distal end of the tibia and fibula. Pain elicited with compression of the tibia and fibula at the mid-calf level is pathognomonic of a syndesmotic or "high ankle" sprain [7,27].

\section{Alternative imaging modalities}

Stress radiographs typically measure the distance from the posterior lip of the distal tibia and the talar dome when anterior stress is applied to the talocrural joint. Used to diagnose mechanical instability of the lateral ligaments, stress radiography is generally painful, unreliable and outdated often requiring the use of local anesthesia. Stress Radiography is often inconclusive due to a high variability in normal values and manual techniques $[1,31,32]$.

Diagnostic ultrasonography is a useful and quick in-office imaging modality that can identify strains, transverse ruptures and longitudinal substance tears of the lateral collateral ligaments and peroneal tendons. Dynamic images in real time help detect functional impairments or joint instability that is not visualized detected with other imaging choices. Convenience of this assessable modality allows for quicker diagnosis and earlier management. Accuracy, however is highly dependent on the operator, the type of ultrasound equipment and level of soft tissue edema. The sensitivity and specificity of US for a lateral ligament injury is $92 \%$ and $64 \%$, respectively. Sensitivity and specificity increase to $100 \%$ and $72 \%$ when US is performed after an inconclusive delayed physical examination $[31,32]$.

Magnetic Resonance Imaging (MRI) is a reliable method for revealing tears of the ATFL and CFL following acute injury. MRI is not indicated in most ankle sprains due to their high incidence, limited accessibility, high cost and false-positive findings. MRI may be extremely beneficial in patients with clinically suspected concomitant injuries, such as osteochondral lesions of the talus, syndesmotic injuries, and peri ankle tendinous tears. MRI is the gold standard imaging modality of ankle ligamentous and intra-articular injuries and when persistent symptoms and chronic ankle instability require further evaluation [31]. The sensitivity and specificity of MRI for ATFL injuries are $92 \%-100 \%$ and $100 \%$, respectively. In comparison with arthroscopy, MRI correctly located the injured portion of the ATFL in 93\% of the cases compared to US 63\%. Overall, MRI is the imaging modality of choice for lateral ankle injuries in elite athletes [32].

\section{Prevention}

Despite an abundance of data on the incidence, diagnosis and management of ankle sprains, the frequency and economic burden of sports related ankle injuries, has steadily increased over time. Moreover, recurrentankle sprains in athletes leading to prolonged downtime, hinderance of sports production and long term sequalae may imply a need for a paradigm shift on how these injuries are evaluated and managed. A shift to a to a more collaborative, interprofessional approach that includes, sports trainers, podiatric and orthopedic specialists, nurse practitioners and physical therapists that will focus on prevention, diagnosis, and recovery. Timely and accurate diagnosis that considers the epidemiology, etiology, risk factors and natural history allows for better understanding and management of the injury. Prevention of a recurrent LAS requires a multidisciplinary team of experts implementing comprehensive treatment protocols that focus on protection, optimal loading, early mobilization, functional rehabilitation and the stabilization of abnormal foot and ankle biomechanics.

Protection, Optimal Loading, Ice, Compression and Elevation (POLICE) combined with functional rehabilitation emphasizes early mobilization, balance, and neuromuscular strengthening. POLICE with a functional rehabilitative program favors a stronger better recovered injury, when compared to the more traditional RICE (Rest, Ice, Compression, Elevation) protocol.

Optimal loading replaces rest and encourages early weight bearing as tolerated. Rest should be of limited duration and restricted to immediately after the trauma. Longer periods of unloading are harmful and may produce adverse changes to tissue biomechanics and morphology. Progressive mechanical loading is more likely to restore the strength and morphological characteristics of collagenous tissue during the healing phase. Early mobilization with accelerated functional rehabilitation is effective after the acute ankle injury. Functional rehabilitation includes early weightbearing, with the aid of external supportive devices such as pneumatic walking boots, lace up ankle braces, and supportive athletic tape. Moreover, early mobilization aligns well with the principles of mechanical therapy and loading 
prompting a cellular response that promotes tissue structural changes conducive to the maturation and remodeling phase of soft tissue repair. Controlled exercises increases the strength and fiber orientation of the lateral ankle ligaments. When appropriately performed, protection and rehabilitation practices enhance the remodeling and final maturation phases of soft tissue healing that typically require between 8 weeks to six months. In turn, the athlete will be ready to return to sport activity with full neuromuscular control, stability and strength [30-32,34,35].

Successful prevention exercise programs emphasize a quick warm up, balance training and proprioceptive drills that will challenge ankle joint position. The warmup includes stretching, strength, agility and balance exercises. Physical therapists and sports trainers employ balance boards, wobble boards or ankle discs. Singlelegand double leg hopping exercise help strengthen the ankle and enhance proprioception, agility, and stability. Some athletes prefer to employ taping and bracing as a substitute to an effective exercise program. Athletes, particularly adolescents, find it easier to consistently tape or brace than to consistently do exercises over the course of weeks and months. Many athletes prefer taping to bracing as they find ankle braces to be restrictive, uncomfortable and bulky resulting in underperformance. Nevertheless, evidence based practice supports combining taping and bracing with a recovery exercise programs in order to achieve the best long term outcomes. Kaminsky concluded that external prophylactic supports and preventive exercise programs are effective for reducing the risk of ankle sprains in both uninjured and previously injured populations. Ankle bracing appears to offer the best outcomes in terms of cost and risk reduction [38]. However, there remains a need for welldesigned, prospective randomized controlled trials relevant to the primary prevention of lateral ankle sprains $[33,38]$.

Core strengthening exercises involving the hip, abdominals and back also aid in the prevention of LAS. The strength and function of the hips and trunk is important for movement and sports performance. Imagine running lightly and abruptly changing direction. A lack of core strength negatively impacts body and hip control, which may lead to an uncontrollable change in the athletes' center of gravity during sudden change in direction. Pushing the body beyond what the foot and ankle can stabilize, may result in an inversion sprain and/or fall.

Improving flexibility of the lower extremity may also play a role in the prevention of initial and recurrent ankle injuries. A balance between strength and flexibility is important. Simple stretching for 30-60 seconds after a light warm-up can help relieve discomfort and promote adequate mobility of the lower leg. The amount of time spent on the activity should be increased gradually over a period of weeks to build both muscle strength and mobility. Cross training by participating in different activities can help build the muscles while avoiding activity adaptation.
Choosing athletic shoes specifically for one's foot type, and biomechanics is important for ankle sprain prevention. Podiatrists may educate and advise the athlete on their foot type and function through biomechanical exams and gait analysis. Shoe selection and custom foot orthoses play a large role in prevention of ankle sprains as stabilizing faulty biomechanics and foot position is crucial in preventing recurrent LAS.

Extensive patient education regarding the natural history of ankle injuries, risk of re injury, long term sequelae, healing timelines and return to activity guidelines must be conveyed to the athlete and coaching staff. Unfortunately, there is a lack of consensus among expert opinion and preferred practice guidelines on return to sports criteria following lateral ankle sprains. Internal pressure from athletic programs to expedite a quick return to sports activity sometimes overrides appropriate medical advice [35-37]. LAS patients often receive care, but an emphasis on rapid return to sport (RTS) may sometimes override the best medical advice.

Wikstrom and Mueller reported a RTS average of 12 days. Moreover, forty four percent (44\%) of intercollegiate athletes seen by healthcare providers for a LAS returned to sport in less than 24 hours [35,36]. In a separate study, comparing initial to recurrent LAS reported the average RTS for an initial injury was 3 days, while recurrent injuries RTS in less than 24 hours. Rapid RTS timelines neglect the biological processes required for adequate healing increasing injury risk and long-term sequelae. Quick Return to play may lead athletes to demonstrate ligamentous laxity, limitations in selfreported function, limited dorsiflexion range of motion, and impaired dynamic postural control. According to Wikstrom RTS decisions for LAS, were derived from anecdotal evidence and practitioner experience and influenced by stakeholders (patients, parents, and coaches) pushing RTS expediency [35-38].

\section{Conclusion}

Every day, thousands of people with ankle sprains limp into Emergency Departments(ED) across the U.S. According to recent data from the National Collegiate Athletic Association (NCAA), the lateral ankle sprain (LAS) is most prevalent in high impact sports like football, basketball, wrestling and ice hockey. The direct and indirect costs related to ankle sprains approximates 4 billion per year and long term sequelae and disability often result when cases are mismanaged. Most LAS do not require surgical intervention, but a comprehensive evaluation, an accurate and timely diagnosis, and an evidence supported treatment plan will help achieve optimum outcomes, quicker return to activity, and a lower risk of recurrence and complications. Ankle sprains in athletes may best be managed by an interprofessional team approach which include, sports trainers, podiatric and orthopedic specialists, nurse practitioners and physical therapists that 
focus on prevention. Patient education by the healthcare team emphasize the importance of stretching and conditioning to minimize the severity of ankle sprain. Athletes should be encouraged to wear proper shoes and use braces or foot orthoses to further protect the ankle, prevent recurrence and enhance performance.

\section{References}

1. Kaminski T, Hertel J, Amendola N, Docherty C, Dolan MG, et al. National Athletic Trainers' Association Position Statement: Conservative Management and Prevention of Ankle Sprains in Athletes. J Athl Train. 2013; 48: 528-545.

PubMed: https://pubmed.ncbi.nlm.nih.gov/23855363/

2. Adams J, Barton E, Collings J. et al. Emergency Medicine. Philadelphia PA: Saunders, Elsevier, Inc. 2008; 897-898.

3. Waterman BR, Owens BP, Davey S, Zacchilii MA, Belmont PJ, Jr., et al. The epidemiology of ankle sprains in the United States. JBJS, 2010; 92: $2279-2284$.

PubMed: https://pubmed.ncbi.nlm.nih.gov/20926721/

4. Holmer P, Sondergaard L, Konradsen L, Nielsen PT, Jørgensen LN Epidemiology of sprains in the lateral ankle and foot. Foot Ankle Int. 1994; 15: 72-74.

PubMed: https://pubmed.ncbi.nlm.nih.gov/7981804/

5. Newsham K. The Ubiquitous Lateral Ankle Sprain: Time to Reconsider Our Management? JNP. 2019; 15: 343-346.

6. Safran MR, Zachazewski JE, Benedetti RS, Bartolozzi AR, Mandelbaum R. Lateral ankle Sprains: a comprehensive review part 2: treatment and rehabilitation with an emphasis on the athlete. Med Sci Sports Exerc. 1999; 31: 438-447.

PubMed: https://pubmed.ncbi.nlm.nih.gov/10416545/

7. Myrick K. Clinical Assessment and Management of Ankle Sprains Ortho Nurs. 2014; 33: 244-248.

PubMed: https://pubmed.ncbi.nlm.nih.gov/25233201/

8. Beynnon BD, Vacek PM, Murphy D, Alosa D, Paller D. First time inversion ankle ligament trauma. Am J Sports Med. 2005; 33: 1485-1491. PubMed: https://pubmed.ncbi.nlm.nih.gov/16009979/

9. Martin R, Davenport T, Paulseth S, Wukich D. Ankle Stability and Movement Coordination Impairments: Ankle Ligament Sprains Clinical Practice Guidelines. J Ortho Sports Phys Ther. 2013; 43: A1-A40. PubMed: https://pubmed.ncbi.nlm.nih.gov/24313720/

10. McKay GD, Goldie PA, Payne WR, Oakes BW. Ankle Injuries in Basketball: Injury Rate and Risk Factors. Br J Sports Med. 2001; 35: 103-108. PubMed: https://pubmed.ncbi.nlm.nih.gov/11273971/

11. DiStefano LJ, Padua DA, Brown CN, Guskiewicz KM. Lower Extremity Kinematics and Ground Reaction Forces after Prophylactic Lace-up Ankle Bracing. J Athletic Train. 2008; 43: 234-341.

PubMed: https://pubmed.ncbi.nlm.nih.gov/18523572/

12. Fong D, Hong $Y$, Chan L, Yeung $P$, et al. A Systematic Review on Ankle Injury and Ankle Sprain in Sports. Sports Med. 2007; 37: 73-94. PubMed: https://pubmed.ncbi.nlm.nih.gov/17190537/

13. Doherty C, Delahunt E, Caulfield B, Hertel J, et al. The incidence and prevalence of ankle sprain injury: A systematic review and meta-analysis of prospective epidemiological studies. Sports Med. 2014; 44: 123-140. PubMed: https://pubmed.ncbi.nlm.nih.gov/24105612/

14. Tropp H, Askling C, Gillquist J. Prevention of ankle sprains. Am J Sports Med. 1985; 13: 259-262.

PubMed: https://pubmed.ncbi.nlm.nih.gov/3927758/

15. Van Rijn RM, Van Os AG, Bernsen RM, LuijsterbVrg PA, Koes BW, et al. What is the clinical course of acute ankle sprains? A systematic literature review. Am J Med. 2008; 121: 324-331.

PubMed: https://pubmed.ncbi.nlm.nih.gov/18374692/
16. Malliaropoulos N, Ntessalen M, Papacostas E, Longo UG, Maffulli N. Reinjury after acute lateral ankle sprains in elite track and field athletes. Am J Sports Med. 2009; 37: 1755-1761.

PubMed: https://pubmed.ncbi.nlm.nih.gov/19617530/

17. Yeung MS, Chan KM, Yuan WH. An epidemiological survey on ankle sprain. Br J Sports Med. 1994; 28: 112-116.

PubMed: https://pubmed.ncbi.nlm.nih.gov/7921910/

18. Hubbard T, Wikstrom EA. Ankle sprain: pathophysiology, predisposing factors, and management strategies. Open Access J Sports Med. 2010; 1: 115-122.

PubMed: https://www.ncbi.nlm.nih.gov/pmc/articles/PMC3781861/

19. Brostroem L. Sprained ankles. Anatomic lesions on recent sprains. Acta Chir Scand. 1964; 128: 483-495.

PubMed: https://pubmed.ncbi.nlm.nih.gov/14227127/

20. Bonnel F, Toullec E, Mabbitt C, Tourné Y, Sofcot. Chronic ankle instability: Biomechanics and pathomechanics of ligaments injury and associated lesions. OTCR. 2010; 96: 424-432.

PubMed: https://pubmed.ncbi.nlm.nih.gov/20493797/

21. Man IO, Morrissey MC. Relationship between ankle-foot swelling and self-assessed function after ankle sprain. Med Sci Sports Exerc. 2005; 37: 360-363.

PubMed: https://pubmed.ncbi.nlm.nih.gov/15741831/

22. Bachmann LM, Kolb E, Koller MT, Steurer J, ter Riet G. Accuracy of Ottawa ankle rules to exclude fractures of the ankle and mid-foot: Systematic Review. BMJ. 2003; 326: 417.

PubMed: https://pubmed.ncbi.nlm.nih.gov/12595378/

23. Bleakley C, O'Connor SR, Tulty MA, Rocke LG, Macauley DC, et al. Effect of accelerated rehabilitation on function after ankle sprain: randomized controlled trial. BMJ. 2010; 340: c1964.

PubMed: https://pubmed.ncbi.nIm.nih.gov/20457737/

24. Braun BL. Effects of ankle sprain in a general clinic population 6 to 18 months after medical evaluation. Arch Fam Med. 1999; 8: 143-148. PubMed: https://pubmed.ncbi.nlm.nih.gov/10101985/

25. Anandacoomarasamy A, Barnsley L. Long term outcomes of inversion ankle injuries. Br J Sports. 2005; 39: e14.

PubMed: https://pubmed.ncbi.nlm.nih.gov/15728682/

26. Karlsson J. Chronic Lateral Instability of the Ankle [master's thesis] Göteborg University; Göteborg, Sweden. 1989; 158.

27. Lynch S. Assessment of the Injured Ankle in the Athlete. J Athl Train. 2002; 37: 406-412.

PubMed: https://pubmed.ncbi.nlm.nih.gov/12937562/

28. Gaebler C, Kukla C, Breitenseher MJ, et al. Diagnosis of lateral ankle ligament injuries: comparison between talar tilt, MRI and operative findings in 112 athletes. Acta Orthop Scand. 1997; 68: 286-290. PubMed: https://pubmed.ncbi.nlm.nih.gov/9246995/

29. Sitler M, Ryan J, Wheeler B, McBride J, Arciero R, et al. The efficacy of a semirigid ankle stabilizer to reduce acute ankle injuries in basketball: a randomized clinical study at West Point. Am J Sports Med. 1994; 22: 454-461.

PubMed: https://pubmed.ncbi.nlm.nih.gov/7943509/

30. Martin R, Davenport T, Fraser J, Sawdon-Bea J, Carcia CR, et al. Ankle Stability and Movement Coordination Impairments: Lateral Ankle Ligament Sprains Revision. J Orthopaedic Sports Physical Ther. 2021; 51: CPG1-CPG80.

PubMed: https://pubmed.ncbi.nlm.nih.gov/33789434/

31. Halabchi F, Hassabi M. Acute ankle sprain in athletes: Clinical aspects and algorithmic approach. World J Orthop. 2020; 11: 534-558. PubMed: https://pubmed.ncbi.nlm.nih.gov/33362991/

32. D'Hooghe P, Cruz F, Alkhelaifi K. Return to Play After a Lateral Ligament Ankle Sprain. Curr Rev Musculoskelet Med. 2020; 13: 281-288. PubMed: https://pubmed.ncbi.nlm.nih.gov/32377961/

33. Green T, Willson G, Martin D, Fallon K. What is the quality of clinical 
practice guidelines for the treatment of acute lateral ankle ligament sprains in adults? A systematic review. BMC Musculoskelet Disord. 2019; 20: 394

PubMed: https://pubmed.ncbi.nlm.nih.gov/31470826/

34. Xue X, Ma T, Li Q, Song Y, Hua Y. Chronic ankle instability is associated with proprioception deficits: A systematic review and meta-analysis. J Sport Health Sci. 2021; 10: 182-191.

PubMed: https://pubmed.ncbi.nlm.nih.gov/33017672/

35. Wikstrom EA, Mueller C, Cain MS. Lack of Consensus on Return-toSport Criteria Following Lateral Ankle Sprain: A Systematic Review of Expert Opinions. J Sport Rehabil. 2019; 29: 231-237.

PubMed: https://pubmed.ncbi.nlm.nih.gov/31141438/
36. Roos KG, Kerr ZY, Mauntel TC, Djoko A, Dompier TP, et al. The epidemiology of lateral ligament complex ankle sprains in national collegiate athletic association sports. Am J Sports Med. 2017; 45: 201-209. PubMed: https://pubmed.ncbi.nlm.nih.gov/27573356/

37. Medina-McKeon JM, Bush HM, Reed A, Whittington A, Uhl TL, et al. Return-to-play probabilities following new versus recurrent ankle sprains in high school athletes. J Sci Med Sport. 2014; 17: 23-28. PubMed: https://pubmed.ncbi.nIm.nih.gov/23689105/

38. Kaminski TW, Needle AR, Delahunt E. Prevention of Lateral Ankle Sprains. J Athl Train. 2019; 54: 650-661.

PubMed: https://pubmed.ncbi.nlm.nih.gov/31116041/ 